\title{
THE MEASURE OF THE INTERSECTION OF ROTATES OF A SET ON THE CIRCLE ${ }^{1}$
}

\author{
WOLFGANG M. SCHMIDT
}

ABSTRACT. Let $S$ be a set of real numbers modulo 1 of Lebe sgue measure less than 1. It is shown that for every $\epsilon>0$ and for large $k$, there exist translates $S+y_{1}, \cdots, S+y_{k}$ of $S$ such that the measure of their intersection is less than $\epsilon^{k}$.

1. Let $U$ be the group of real numbers modulo 1 , and $S$ a subset of Lebesgue measure $\mu(S)<1$. Given real numbers $y_{1}, \cdots, y_{k}$, write $\mu\left(y_{1}, \cdots, y_{k}\right)$ for the measure of the intersection of the $k$ translates $S+$ $y_{1}, S+y_{2}, \cdots, S+y_{k}$. Finally, denote by $\phi(k)$ the infimum of $\mu\left(y_{1}, \cdots, y_{k}\right)$ over all $k$-tuples $y_{1}, \cdots, y_{k}$. Erdös, Rubel and Spencer ${ }^{2}$ had conjectured that

$$
\lim _{k \rightarrow \infty} \phi(k)^{1 / k}=0 .
$$

In the present note we shall prove this conjecture.

The convergence expressed by (1) is not uniform with respect to the sets S. In fact, it can be shown that for $0<\alpha<1, \epsilon>0$ and $k \geq 1$, there exist sets $S$ with $\mu(S)=\alpha$ and $\phi(k)^{1 / k}>a-\epsilon$.

2. Since $\mu(S)<1$, the set $S$ is contained in a countable union of intervals whose total measure is less than 1 . In fact, this is true even with intervals of the type $a \leq x<b^{3}$ with rational endpoints $a, b$. Hence we may assume that $S$ itself is a countable union of such intervals.

Using the easily established relation

$$
\int_{U} \mu\left(y_{1}, \cdots, y_{m}, z_{1}+x, \cdots, z_{n}+x\right) d x=\mu\left(y_{1}, \cdots, y_{m}\right) \mu\left(z_{1}, \cdots, z_{n}\right),
$$

Received by the editors October 6, 1972 and, in revised form, January 11, 1974. AMS (MOS) subject classifications (1970). Primary 10F 40; Secondary 28-00, $43 \mathrm{~A} 05$.

Key words and phrases. Numbers modulo 1, measure, translates.

1 Research supported by NSF GP-33026X.

2 P. Erdös, L. A. Rubel and J. H. Spencer, in the problem collection of the 1972 number theory conference in Colorado.

3 We are considering intervals modulo 1 . Hence if $\{x\}$ denotes the fractional part of $x$, the interval $a \leq x<b$ consists of numbers $x$ modulo 1 with $\{x-a\}<$ $\{x-b\}$. 
one sees that $\phi(m+n) \leq \phi(m) \phi(n)$. Hence if $t$ is any positive integer, we have $\phi(j t) \leq \phi(t)^{j}(j=1,2, \cdots)$, and if $k$ is a large integer with $j t<k \leq$ $(j+1) t$, then $\phi(k) \leq \phi(j t) \phi(k-j t) \leq \phi(j t) \leq \phi(t)^{j}$ and

$$
\phi(k)^{1 / k} \leq \phi(t)^{j / k} \leq \phi(t)^{(1 / t)-(1 / k)} .
$$

Therefore the limit superior of $\phi(k)^{1 / k}$ as $k \rightarrow \infty$ cannot exceed $\phi(t)^{1 / t}$. Thus in order to prove (1), it will suffice to show that for every $\epsilon>0$ there is an integer $t$ with

$$
\phi(t)^{1 / t}<\epsilon
$$

3. Write $\mu(S)=\mu$, and choose $\delta>0$ so small that

$$
2 \delta<1-\mu \text { and }(\delta /(1-\mu-\delta))^{1-\mu-\delta}<\epsilon .
$$

We may write $S=S_{1} \cup S_{2}$, where $S_{1}$ is a finite union of intervals $a \leq x<b$ with rational endpoints, and where $\mu\left(S_{2}\right)<\delta$.

Let $r$ be a common denominator of the endpoints of the intervals contributing to $s_{1}$. Choose an integer $s$ with $s>1 / \delta$, and put

$$
t \times r s, \quad \nu=1 / t .
$$

Let $\chi(x)$ be the characteristic function of $S$, and write

$$
I_{\nu}(y)=\int_{y}^{y+\nu} \chi(x) d x
$$

Lemma. The function

$$
J_{\nu}(z)=I_{\nu}(\dot{z}+\nu) I_{\nu}(z+2 \nu) \cdots I_{\nu}(z+t \nu)
$$

satisfies $J_{\nu}(z) \leq(\epsilon \nu)^{t}$.

To prove the Lemma, we observe that $S_{1}$ consists of a finite number (in fact less than $r$ ) intervals $E$ of the type $(u / r) \leq x<(u+1) / r$ with integral $u$. For each such interval $E$ contained in $S_{1}$, let $E^{\prime}$ be the enlarged interval $(u / r)-(1 / t) \leq x<(u+1) / r$. Let $S_{1}^{\prime}$ be the union of the intervals $E^{\prime}$ so obtained. It is clear that

$$
\text { if } x+w \in S_{1} \text { with } 0 \leq w \leq \nu \text {, then } x \in S_{1}^{\prime} \text {. }
$$

For each interval $E$ above we have $\mu\left(E^{\prime}\right)=\mu(E)+(1 / t)$, and hence we have $\mu\left(S_{1}^{\prime}\right)<\mu\left(S_{1}\right)+(r / t)=\mu\left(S_{1}\right)+(1 / s)<\mu(S)+\delta=\mu+\delta$. Now $S_{1}^{\prime}$ is a disjoint union of intervals $(v / t) \leq x<(v+1) / t$ with integral $v$. If, say, it is a disjoint union of $p$ such intervals, then $\mu\left(S_{1}^{\prime}\right)=p / t$ and hence

$$
p=t \mu\left(S_{1}^{\prime}\right)<t(\mu+\delta) \text {. }
$$


Exactly $q=t-p$ of the numbers $z+\nu, z+2 \nu, \cdots, z+t \nu$ lie outside $S_{1}^{\prime}$; let these be the numbers $z+m_{1} \nu, z+m_{2} \nu, \cdots, z+m_{q} \nu$. Since each integral $I_{\nu}(y)$ is always $\leq \nu$, we have

$$
J_{\nu}(z) \leq \nu^{p} I_{\nu}\left(z+m_{1} \nu\right) \cdots I_{\nu}\left(z+m_{q} \nu\right) .
$$

Now $I_{\nu}\left(z+m_{i} \nu\right)$ is the integral of $\chi(x)$ over the interval $z+m_{i} \nu \leq x<z+$ $\left(m_{i}+1\right) \nu(i=1, \cdots, d)$. These intervals are disjoint from each other.

Furthermore, since $z+m_{i} \nu \notin S_{1}^{\prime}$, (5) implies that these intervals are disjoint from $S_{1}$. Therefore if $\bar{S}_{1}$ is the complement of $S_{1}$, we have

$$
I_{\nu}\left(z+m_{1} \nu\right)+\cdots+I_{\nu}\left(z+m_{q} \nu\right) \leq \int_{S_{1}} \chi(x) d x \leq \mu\left(S_{2}\right)<\delta .
$$

By the arithmetic-geometric in equality, the product of the $q$ integrals on the left is $<(\delta / q)^{q}$, and (7) yields

$$
J_{\nu}(z)<\nu^{p}(\delta / q)^{q}=\nu^{t}(\delta t / q)^{q} .
$$

From (6) we have $q=t-p>t(1-\mu-\delta)$, whence

$$
(\delta t / q)^{q}<(\delta /(1-\mu-\delta))^{q}<(\delta /(1-\mu-\delta))^{t(1-\mu-\delta)}<\epsilon^{t}
$$

by (3), and the Lemma is proved.

4. The desired inequality (2) follows at once from the Lemma by observing that

$$
\begin{aligned}
\phi(t) & \leq \nu^{-t} \int_{\nu}^{2 \nu} d y_{1} \cdots \int_{t \nu}^{(t+1) \nu} d y_{t} \mu\left(-y_{1}, \cdots,-y_{t}\right) \\
& =\nu^{-t} \int_{U} d x \int_{\nu}^{2 \nu} d y_{1} \cdots \int_{t \nu}^{(t+1) \nu} d y_{t} \chi^{\left(x+y_{1}\right) \cdots \chi\left(x+y_{t}\right)} \\
& =\nu^{-t} \int_{U} d x I_{\nu}(x+\nu) I_{\nu}(x+2 \nu) \cdots I_{\nu}(x+t \nu) \\
& =\nu^{-t} \int_{U} J_{\nu}(x) d x<\nu^{-t}(\epsilon \nu)^{t}=\epsilon^{t} .
\end{aligned}
$$

DEPARTMENT OF MATHEMATICS, UNIVERSITY OF COLORADO, BOULDER, COLORADO 80302 